\title{
Caracterización electroquímica de películas de óxido de zinc obtenidas por anodizado en medio alcalino
}

\author{
Electrochemical characterization of zinc oxide \\ films obtained by anodizing in \\ alkaline medium
}

\author{
Daiana Jennifer Díaz ${ }^{1}$, Gustavo Raúl Kramer ${ }^{1,2}$, Hernán Darío Traid ${ }^{1,2}$, \\ Claudia Marcela Méndez ${ }^{1,2}$, Alicia Esther Ares ${ }^{1,2}$
}

\author{
${ }^{1}$ Facultad de Ciencias Exactas, Químicas y Naturales (FCEQyN), Universidad Nacional de Misiones (UNaM), Félix de \\ Azara 1552, Posadas, Misiones, Argentina. \\ e-mail: daianajenniferdiaz@gmail.com \\ ${ }^{2}$ IMAM (CONICET-UNaM), Posadas, Misiones, Argentina. \\ e-mail: grkramer@fceqyn.unam.edu.ar, traidhernan@gmail.com, cmendez@fceqyn.unam.edu.ar, aa- \\ res@fceqyn.unam.edu.ar
}

\section{RESUMEN}

El óxido de Zinc es un material de particular interés a causa de sus propiedades ópticas y eléctricas únicas, que son exploradas para muchas aplicaciones en optoelectrónica y nanoelectrónica; y que puede ser sintetizado de manera económica por la técnica de anodización. En este trabajo se empleó la técnica de anodizado potenciostático en solución diluida de $\mathrm{NaOH}$, a temperatura ambiente, para la obtención de películas anódicas de $\mathrm{ZnO}$. Las propiedades morfológicas y estructurales de los anodizados obtenidos a diferentes voltajes, y de las películas sometidas a tratamiento térmico, se caracterizaron por microscopía electrónica de barrido (SEM) encontrándose que las películas son compactas, de morfología heterogénea, y que las dimensiones de las partículas son proporcionales al potencial aplicado. Asimismo, se realizaron estudios electroquímicos mediante la aplicación de la técnica de Espectroscopía de Impedancia Electroquímica (EIS) para evaluar las propiedades electroquímicas de las películas. Se demostró que las películas de ZnO presentan un comportamiento resistivo y capacitivo, este último se asocia a la presencia de un elemento de fase constante con coeficiente de identidad (n) definido levemente menor a la unidad. Los espesores de las películas se determinaron ajustando los resultados experimentales obtenidos por EIS a un circuito equivalente considerando el elemento de fase constante como capacitor ideal. En base a los parámetros analizados se concluyó que al aplicar potenciales más elevados se forman capas de mayor espesor y menor resistencia, en tanto que a potenciales menores las capas tienden a ser más delgadas y resistivas. Por otro lado, se demostró que el tratamiento térmico aplicado provocó una disminución de la resistencia eléctrica de las películas, y un aumento de la capacitancia del sistema, con la consecuente disminución del espesor, respecto a las películas sin tratamiento térmico, pero no se encontraron diferencias en las propiedades electroquímicas entre las películas tratadas térmicamente.

Palabras clave: Óxido de Zinc, Anodizado, Espectroscopía de Impedancia Electroquímica.

\section{ABSTRACT}

Zinc Oxide is a material of particular interest because of its unique optical and electrical properties, which are explored for many applications in optoelectronics and nanoelectronics; and it that can be synthesized economically by the technique of anodization. In this work, the potentiostatic anodization technique was used in dilute $\mathrm{NaOH}$ solution, at room temperature, to obtain $\mathrm{ZnO}$ anodic films. The morphological and structural properties of the anodized films obtained at different voltages and of the films subjected to heat treatment were characterized by scanning electron microscopy (SEM) and the films were found to be compact, of heterogeneous morphology, and that the particle size was proportional to the applied potential. In addition, electrochemical studies were carried out by applying the Electrochemical Impedance Spectroscopy (EIS) technique to evaluate the electrochemical properties of the films. It was demonstrated that the $\mathrm{ZnO}$ films presented 
a resistive and capacitive behavior, the latter being associated to the presence of a constant phase element with identity coefficient (n) defined slightly lower than unity. The thicknesses of the films were determined by adjusting the experimental results obtained by EIS to an equivalent circuit considering the constant phase element as an ideal capacitor. Based on the parameters analyzed it was concluded that larger layers were applied with greater thickness and less resistance, while at lower potentials the layers tended to be thinner and resistive. On the other hand, it was demonstrated that the applied heat treatment caused a decrease in the electrical resistance of the films, and an increase in the capacitance of the system, with its consequent decrease in thickness, with respect to films without heat treatment, but no differences were found in the electrochemical properties between the thermally treated films.

Keywords: Zinc Oxide, Anodized, Electrochemical Impedance Spectroscopy.

\section{INTRODUCCIÓN}

Entre los materiales semiconductores utilizados en la actualidad, el Óxido de Zinc (ZnO) ocupa un lugar destacado en las investigaciones recientes a causa de sus propiedades ópticas y eléctricas únicas. Su energía de banda prohibida (Gap) de 3,37 eV, su alta conductividad y su elevada energía de enlace de los excitones igual a $60 \mathrm{meV}$ a temperatura ambiente, le brindan las características necesarias para su potencial aplicación en dispositivos optoelectrónicos y nanoelectrónicos [1,2]. Sin embargo, el interés comercial de este material no solo radica en sus propiedades, sino que además presenta otras ventajas tales como su bajo costo, un impacto ambiental poco significativo ya que se lo considera como un material amigable con el medio ambiente, y su proceso de fabricación relativamente sencillo [1].

Es posible producir películas policristalinas delgadas de $\mathrm{ZnO}$ por diversos métodos, entre los cuales se destacan las técnicas de deposición electroquímica, los procesos sol-gel, los procesos de transporte en fase de vapor, la oxidación anódica, entre otras.

El anodizado es un proceso electroquímico mediante el cual se producen películas de óxidos adhesivas y uniformes en la superficie de un sustrato metálico, la naturaleza de dichas películas dependerán exclusivamente de los parámetros de síntesis, es decir, las condiciones operacionales tales como la concentración y tipo de electrolito utilizado, el voltaje (o densidad de corriente) aplicado, el tiempo de oxidación, la temperatura de trabajo e incluso la estructura granular y preparación superficial del sustrato tendrán una influencia directa en las propiedades de las películas de óxido obtenidas [3].

Es factible producir películas de $\mathrm{ZnO}$ mediante el anodizado de un sustrato de Zinc de alta pureza en un medio electrolítico alcalino. La síntesis de nanopartículas de $\mathrm{ZnO}$ ocurre merced a la ionización del sustrato sumergido en la disolución acuosa a $\mathrm{Zn}^{2+}$ luego de que se aplica un potencial. Se cree que luego de que se enciende la fuente, se conduce una elevada densidad de corriente que produce la hidrólisis de las moléculas de agua a iones $\mathrm{H}^{+}$e $\mathrm{OH}^{-}$, los iones $\mathrm{OH}^{-}$migran hasta la superficie del Zinc dando lugar a la formación de un ión complejo $\mathrm{Zn}(\mathrm{OH})_{2}{ }^{-4}$, que luego precipita sobre la superficie del ánodo cuando su concentración excede su producto de solubilidad, finalmente el precipitado se deshidrata dando lugar a la aparición de las partículas de $\mathrm{ZnO}$ que constituyen una película delgada de óxido en la superficie del sustrato [4-6].

Basándose en numerosos estudios, GOH, ADNAN Y FARRUKH [6] reportaron en sus trabajos de investigación el siguiente mecanismo de reacción para la formación de $\mathrm{ZnO}$.

Ánodo

$Z n \rightarrow Z n^{+2}+2 e^{-}$

$\mathrm{Zn}^{+2}+4 \mathrm{OH}^{-} \rightarrow \mathrm{Zn}(\mathrm{OH})_{2}^{-4}(a q)$

$\mathrm{Zn}(\mathrm{OH})_{2}{ }^{-4}(\mathrm{aq}) \rightarrow \mathrm{Zn}(\mathrm{OH})_{2}(s)+2 \mathrm{OH}$

$2 \mathrm{Zn}(\mathrm{OH})_{2}(s) \rightarrow 2 \mathrm{ZnO}(s)+2 \mathrm{H}_{2} \mathrm{O}(a q)$

Cátodo

$2 \mathrm{H}^{+}+2 e^{-} \rightarrow \mathrm{H}_{2}$ (Reducción)

Si bien la técnica de anodizado permite la obtención de películas uniformes y homogéneas en toda la 
extensión del área sometida al proceso de oxidación, es posible que sea necesario incrementar las características de las películas obtenidas para mejorar su desempeño en ciertas aplicaciones, esto se consigue aplicando tratamientos superficiales a los óxidos obtenidos, para mejorar y controlar su cristalinidad.

Los tratamientos térmicos aplicados a los recubrimientos de $\mathrm{ZnO}$ influyen significativamente en su resistividad eléctrica y sus propiedades ópticas y estructurales, debido a la disminución de los defectos en su estructura cristalina $[7,8]$. La temperatura de recocido es quizá el factor más importante que determinará las propiedades de las películas tratadas, ya que a temperaturas muy elevadas se espera que ocurra un reordenamiento estructural, mientras que a temperaturas a temperaturas superiores a $125^{\circ} \mathrm{C}$ y cercanas al punto de fusión del Zinc, el fenómeno de mayor importancia es la liberación de agua del $\mathrm{ZnOH}_{2}$ remanente en la superficie, tal como se observa en la ecuación (6) $[8,9]$

$$
\mathrm{Zn}(\mathrm{OH})_{2}(s) \rightarrow \mathrm{ZnO}(s)+\mathrm{H}_{2} \mathrm{O} \text { (recocido a } 250^{\circ} \mathrm{C} \text { ) }
$$

La evaluación de recubrimientos por Espectroscopia de Impedancia Electroquímica (por sus siglas en inglés $E I S$ ) enfatiza el cálculo de diversos parámetros como la resistencia y capacitancia de la capa de óxido, mediante la aproximación a circuitos eléctricos equivalentes, y provee información útil acerca del desempeño del recubrimiento y la velocidad de los procesos que allí ocurren [10].

En el presente trabajo se sintetizan películas delgadas de $\mathrm{ZnO}$ empleando la técnica de anodizado potenciostático y se evalúa la influencia del potencial aplicado y el efecto del tratamiento térmico en las propiedades electroquímicas de las mismas.

\section{MATERIALES Y MÉTODOS}

Se utilizaron como sustratos barras de Zinc de alta pureza (99,99\%) de $20 \mathrm{~mm}$ de largo, $7.5 \mathrm{~mm}$ de ancho y 5 $\mathrm{mm}$ de espesor, con estructura granular equiaxial. Previo al anodizado, las probetas fueron pulidas mecánicamente hasta terminación alúmina lubricada con agua, y luego fueron aisladas con un material polimérico dejando disponible para la oxidación $1 \mathrm{~cm}^{2}$ del material, finalmente, las probetas se desengrasaron con etanol. La obtención del $\mathrm{ZnO}$ sobre el sustrato se realizó en una celda electroquímica, empleando un electrodo de Platino como cátodo y colocando la probeta de Zinc en el lugar del ánodo (Figura 1). Los anodizados se realizaron haciendo circular corriente continua entre el ánodo y el cátodo inmersos en una solución electrolítica de $\mathrm{NaOH} 0,1 \mathrm{M}$, a temperatura ambiente, sin agitación, en condiciones potenciostáticas, trabajando con potenciales entre $10 \mathrm{~V}$ y $40 \mathrm{~V}$, con un tiempo de anodizado de 60 minutos [4].

Posteriormente, las películas de $\mathrm{ZnO}$ obtenidas fueron sometidas a un tratamiento térmico a $250^{\circ} \mathrm{C}$ durante dos horas [11]. Las propiedades de las películas obtenidas se determinaron antes y después del tratamiento térmico aplicado por Espectroscopía de Impedancia Electroquímica (EIS) y Microscopía Electrónica de Barrido (SEM).

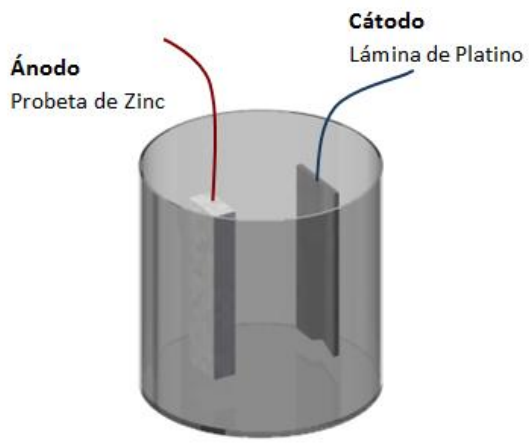

Figura 1: Esquema simplificado de la celda de dos electrodos utilizada para los anodizados.

La técnica de EIS permite deducir el comportamiento electroquímico de la interfaz electrodo-solución, suministrando información relevante para la estimación del desempeño de los recubrimientos.

La impedancia electroquímica se mide aplicando un estímulo de potencial sinusoidal de baja amplitud a una celda electroquímica realizándose un barrido discreto de frecuencias; se detecta la respuesta de corriente en la celda (que será sinusoidal, pero desfasada y con amplitud proporcional al módulo de la impedancia) y 
se despliegan los diagramas de impedancia: Diagrama de Nyquist y Diagrama de Bode (módulo de la impedancia $\mathrm{Z}$ y la diferencia de fase $\varphi$ en función del logaritmo de frecuencia). Los espectros de impedancia se pueden analizar mediante el ajuste de circuitos eléctricos equivalentes cuyos componentes suelen ser elementos eléctricos comunes tales como resistencias, condensadores e inductores, que tienen una base en la electroquímica física del sistema [12]. En la Figura 2 se muestra un circuito equivalente simple utilizado con frecuencia para representar una interface electrodo con recubrimiento compacto-electrolito, donde $R_{\text {sol }}$ representa la resistencia del electrolito, en el cual la migración de iones presenta un comportamiento óhmico, $\mathrm{R}_{\text {oxido }}$ es la resistencia asociada al recubrimiento y CPE es un elemento de fase constante que se define en función del valor que adquiere el coeficiente de identidad "n" durante el ajuste del circuito.

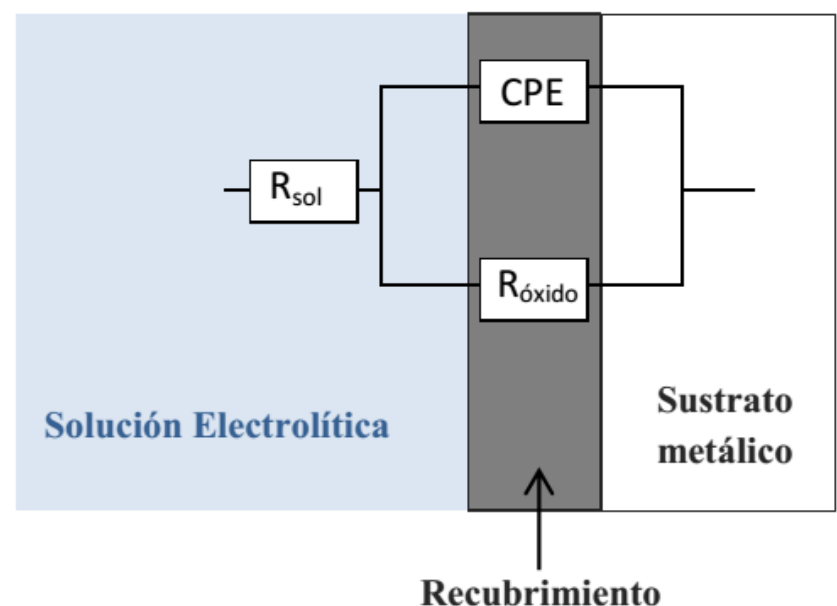

Figura 2: Circuito eléctrico equivalente, representación en una interfase electrodo con recubrimiento compacto-solución.

En el presente trabajo, las medidas de Espectroscopía de Impedancia Electroquímica (EIS) realizadas a los anodizados con y sin tratamiento térmico se llevaron a cabo mediante la utilización de un equipo Gamry FAS1 Femtostat ${ }^{\circledR}$, empleando una celda de tres electrodos. Se utilizó un electrodo de Calomel saturado como electrodo de referencia y un electrodo de Platino como contra electrodo. El electrolito utilizado fue una solución $0,1 \mathrm{M}$ de $\mathrm{NaOH}$ [13]. El electrodo de trabajo fue estabilizado por 60 minutos al Potencial de Circuito Abierto, antes de cada medida. Los datos obtenidos en cada medida se ajustaron a un circuito equivalente para determinar los parámetros electroquímicos significativos.

La morfología de los recubrimientos se observó mediante Microscopía Electrónica de Barrido (por sus siglas en inglés SEM), empleando un microscopio Carl Zeiss Supra $40^{\circledR}$ de la Universidad de Buenos Aires, Argentina.

\section{RESULTADOS}

Al encender la fuente, se forma un campo eléctrico en la superficie de la barra de Zinc que conduce una gran densidad de corriente en los conductores dando lugar a las reacciones de oxidación y reducción. A medida que las reacciones evolucionan, la corriente experimenta variaciones que dependen de las características de la película que se está sintetizando, en la Figura 3 se presenta la variación de la densidad de corriente durante el proceso de oxidación anódica del Zinc a diferentes valores de potencial aplicado, en la misma se puede apreciar un rápido decrecimiento de la densidad de corriente hasta un valor estable que depende del voltaje aplicado. 


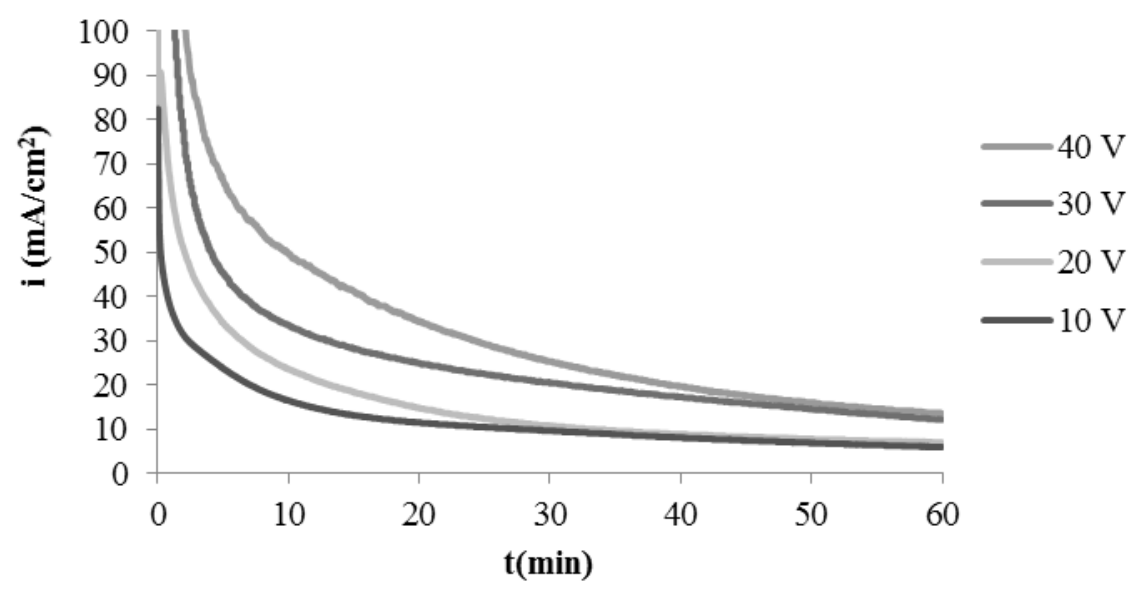

Figura 3: Gráfico de la densidad de corriente (i) en función del tiempo (t) obtenido durante la formación de ZnO.

La disminución en la densidad de corriente se asocia a la formación de una película delgada de naturaleza compacta y resistiva. Para todos los potenciales de trabajo se observa que la corriente disminuye exponencialmente hasta alcanzar un valor estable, esto ocurre porque a medida que transcurre el proceso de oxidación las nuevas partículas de $\mathrm{ZnO}$ sintetizadas se depositan sobre las primeras aumentando progresivamente el espesor de la película de óxido, y en consecuencia su resistividad eléctrica; sin embargo, el aumento del espesor de las películas es limitado, es decir, se alcanza un valor de corriente para el cual la velocidad de formación de $\mathrm{ZnO}$ y la velocidad de disolución del mismo son iguales [14], y el espesor de la película se mantiene constante.

La naturaleza compacta de las películas se puede apreciar con mayor detalle en las micrografías presentadas en la Figura 4. Se observa que los recubrimientos están constituidos de partículas de $\mathrm{ZnO}$ cuyos diámetros son proporcionales al voltaje aplicado, es decir, que a mayor voltaje se observan partículas de dimensiones mayores respecto a las obtenidas a voltajes inferiores. Por otra parte, al aumentar el potencial de trabajo, las partículas de $\mathrm{ZnO}$ tienden a formar aglomerados, y estos arreglos desordenados dan lugar a la formación de estructuras de morfologías poco homogéneas. Se cree también, que a voltajes superiores, los incrementos locales de temperatura producidos por efecto Joule, producen la disolución selectiva de los óxidos, contribuyendo a la falta de homogeneidad en las estructuras de las películas obtenidas [4], este efecto es más significativo en ausencia de agitación del medio electrolítico (ver Figura 4.a y Figura 4.d)

El tratamiento térmico aplicado a las películas contribuye a homogeneizar las morfologías de las estructuras. En la Figura 4.c se observa que la película sometida a tratamiento térmico presenta una compactación mayor respecto a la película obtenida en las mismas condiciones sin aplicar un tratamiento térmico (Figura 4.b), y además tiene un aspecto más homogéneo. 
(a)
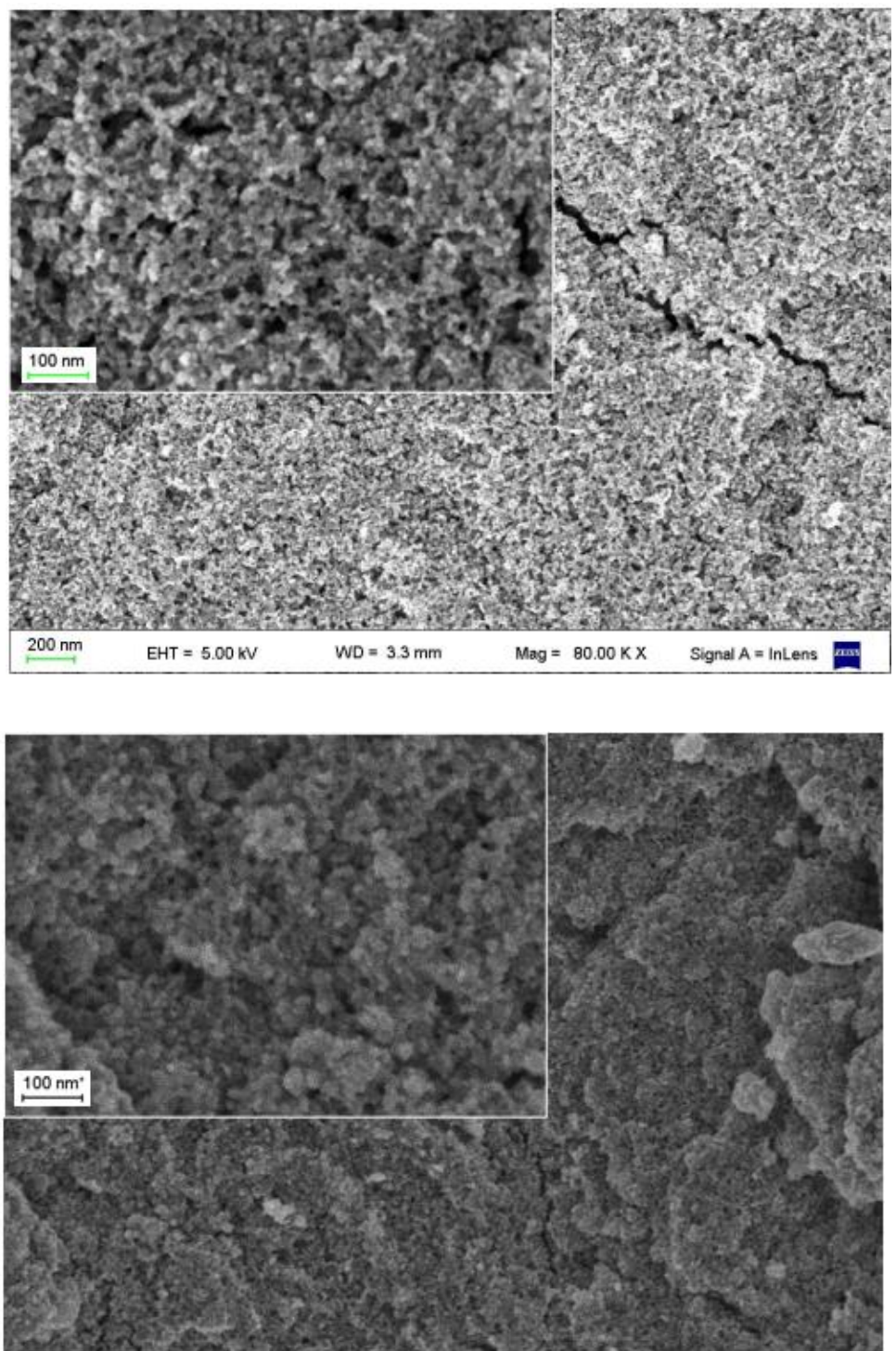

(b)

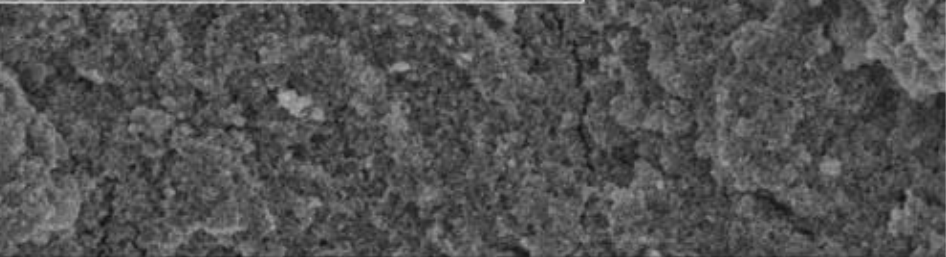

b)

$E H T=3.00 \mathrm{kV}$

$W D=4.2 \mathrm{~mm}$

$\operatorname{Mag}=80.00 \mathrm{KX}$

Signal $A=$ InLens

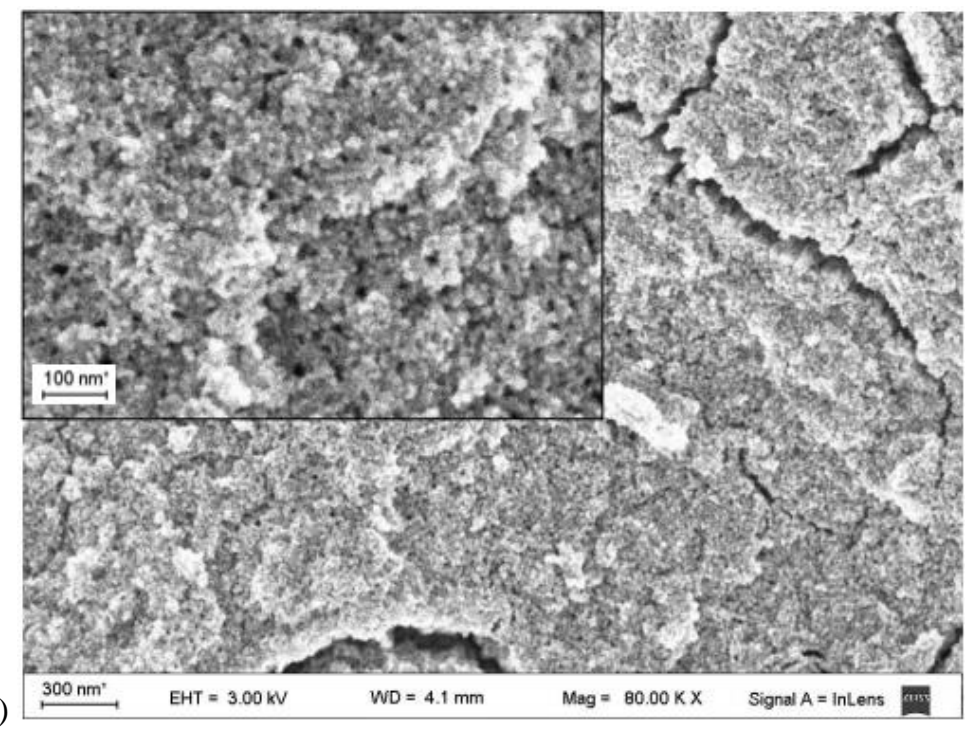




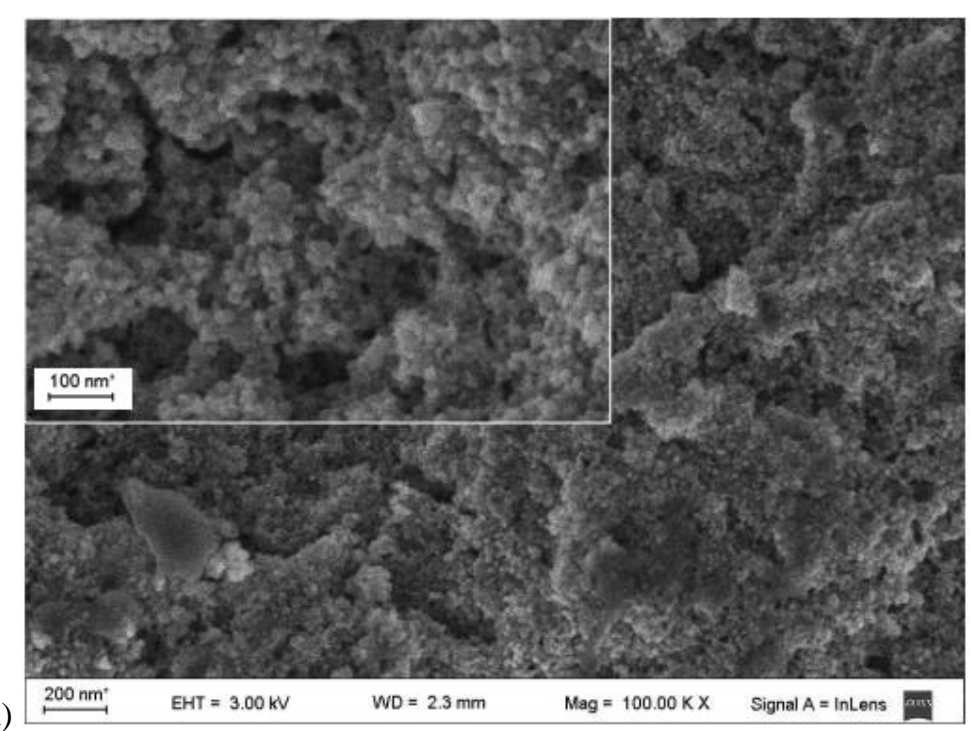

Figura 4: Imágenes $\mathrm{SEM}$ de las películas de $\mathrm{ZnO}$ anodizadas en solución $0,1 \mathrm{M} \mathrm{NaOH}$ por 60 minutos, a: (a) $10 \mathrm{~V}$ sin tratamiento térmico, (b) $30 \mathrm{~V}$ sin tratamiento térmico, (c) $30 \mathrm{~V}$ con tratamiento térmico y (d) $40 \mathrm{~V}$ sin tratamiento térmico.

\section{DISCUSIÓN}

\subsection{Estudios electroquímicos. Espesor de las películas anódicas}

Para evaluar las características electroquímicas de las películas de $\mathrm{ZnO}$ obtenidas sin tratamiento térmico y con tratamiento térmico, se empleó la técnica de Espectroscopía de Impedancia Electroquímica (EIS). En la Figura 5, 6 y 7, se muestran los resultados de las medidas de EIS llevadas a cabo en un medio electrolítico constituido por una solución $0,1 \mathrm{M}$ de $\mathrm{NaOH}$, para varios especímenes de Zinc anodizados sin y con tratamiento térmico, respectivamente.

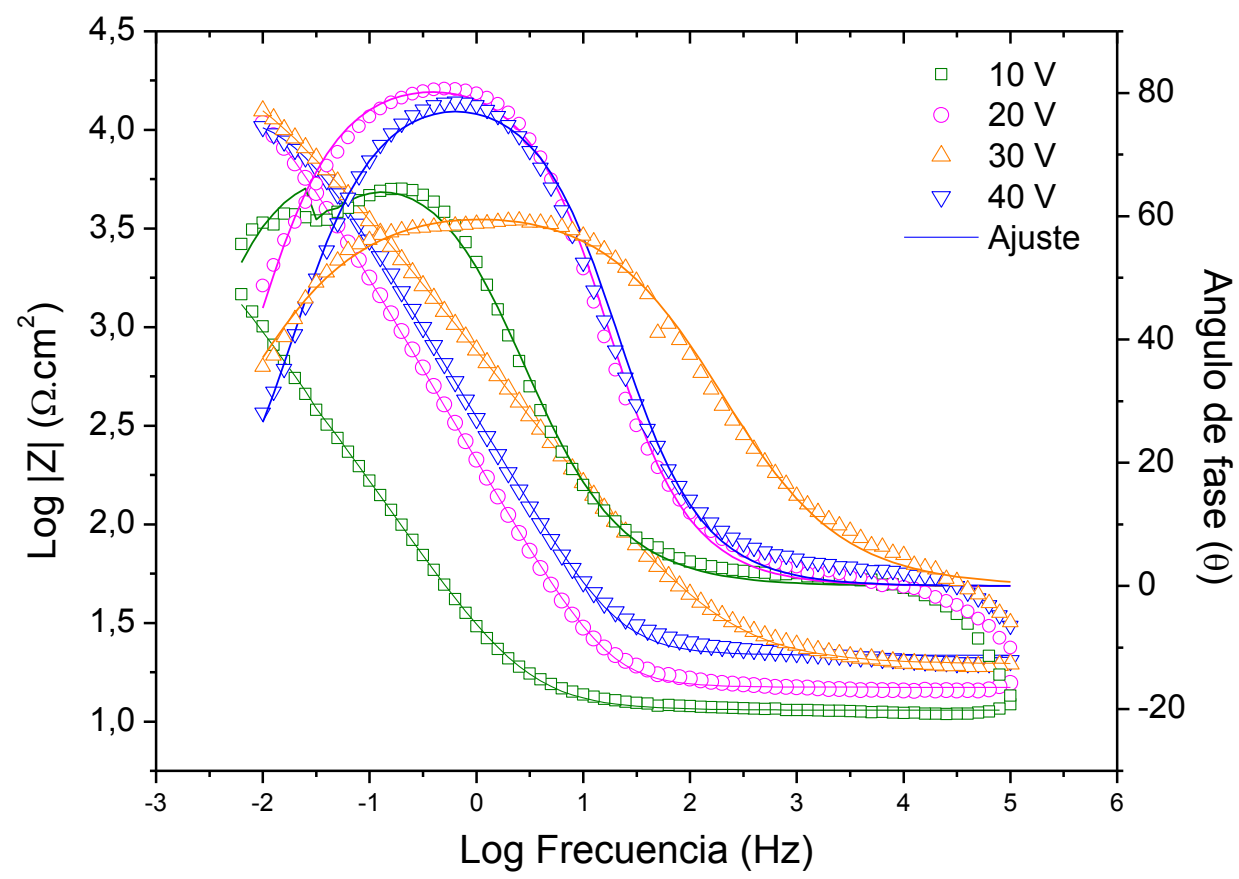

Figura 5: Diagrama de BODE para las experiencias a $10 \mathrm{~V}, 20 \mathrm{~V}, 30 \mathrm{~V}$ y $40 \mathrm{~V}$, sin tratamiento térmico. 


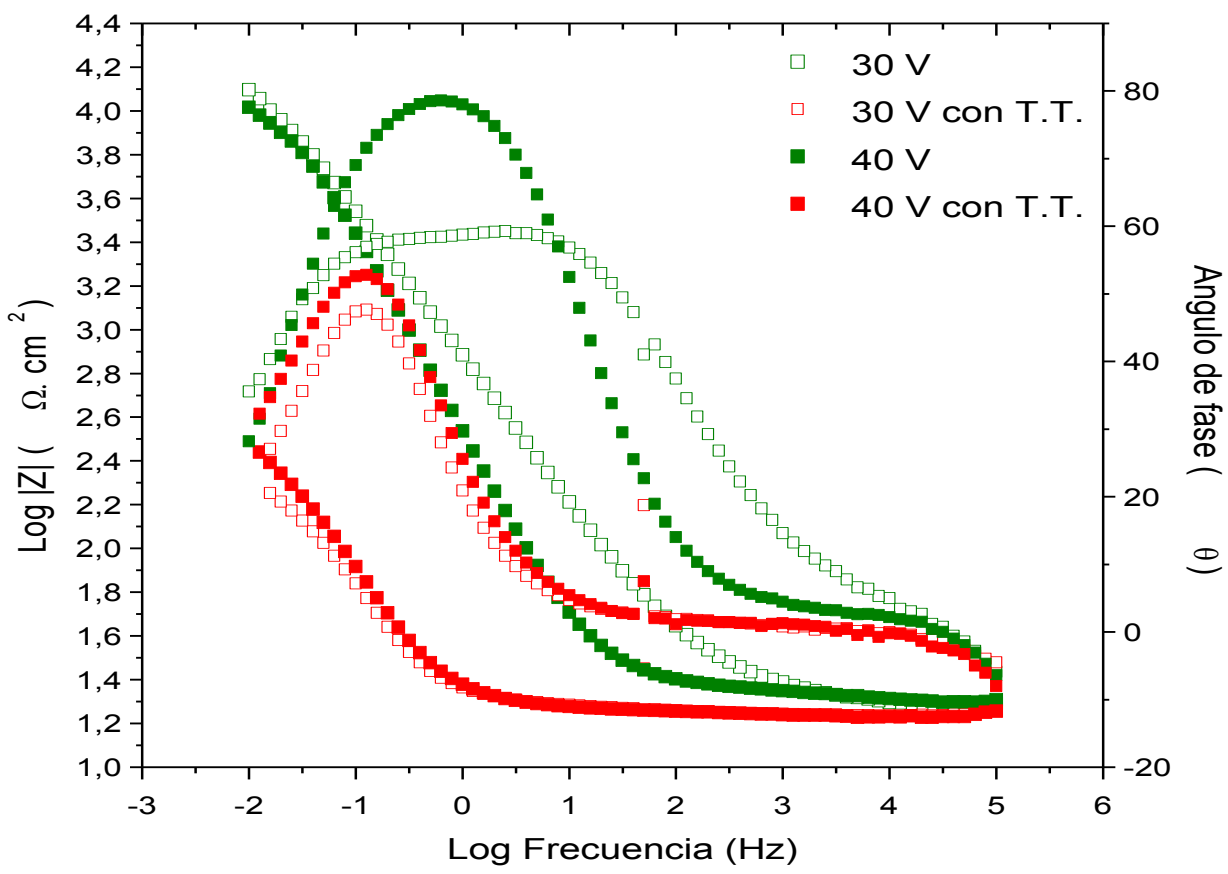

Figura 6: Diagrama de BODE para las experiencias a $40 \mathrm{~V}$ y 30V, sin tratamiento térmico, y con tratamiento térmico.

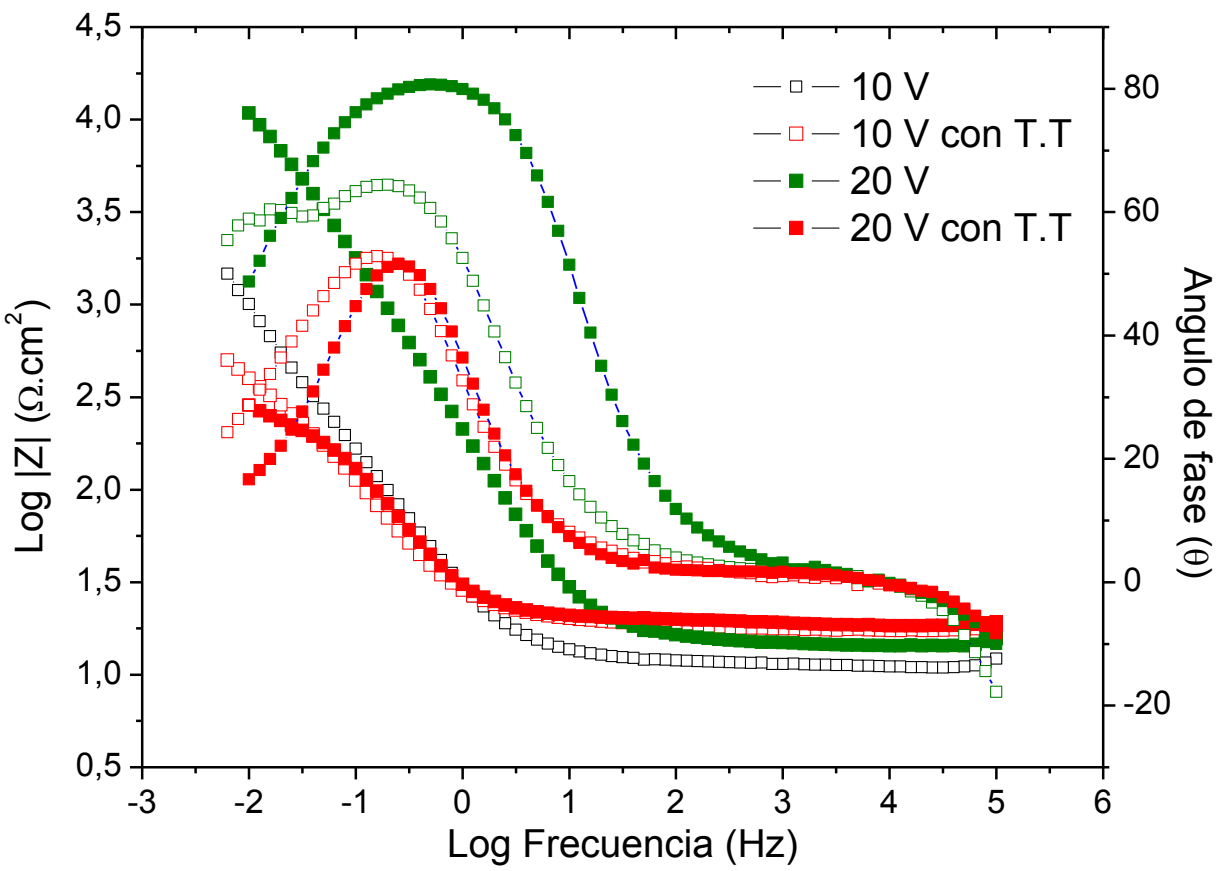

Figura 7: Diagrama de BODE para las experiencias a $10 \mathrm{~V}$ y 20V, sin tratamiento térmico, y con tratamiento térmico.

Los datos obtenidos por este método se ajustaron a un circuito equivalente simple, representado en la Figura 8. El mismo está constituido por un elemento de fase constante (CPE) que se encuentra en paralelo con la resistencia $R_{\mathrm{ox}}$ (resistencia asociada a la película de óxido formada), y éstos a su vez, se encuentran en serie con la resistencia asociada al medio electrolítico o también denominada resistencia óhmica $\left(\mathrm{R}_{\text {sol }}\right)$. En la Tabla 1 se presentan los valores de los elementos pasivos, resultantes de la aproximación a circuitos equivalentes. Como puede preciarse, en todos los casos el valor del coeficiente de identidad o exponente empirico "n", que define la naturaleza del elemento de fase constante (CPE) es superior al valor de 0.5 , atribuible a un comportamiento capacitivo. 
Tabla 1: Valores de los elementos constituyentes de los circuitos eléctricos equivalentes obtenidos por EIS.

\begin{tabular}{|c|c|c|c|c|}
\hline POTENCIAL & $10 \mathrm{~V}$ & $20 \mathrm{~V}$ & $30 \mathrm{~V}$ & $40 \mathrm{~V}$ \\
\hline \multicolumn{5}{|c|}{ SIN TRATAMIENTO TÉRMICO } \\
\hline $\mathrm{R}_{\text {solución }}\left(\Omega \cdot \mathrm{cm}^{2}\right)$ & 11,4 & 11,9 & 19,6 & 21,7 \\
\hline $\mathrm{R}_{\text {oxido }}\left(\Omega . \mathrm{cm}^{2}\right)$ & $1,58 \times 10^{+3}$ & $1,71 \times 10^{+4}$ & $2,61 \times 10^{+4}$ & $1,24 \times 10^{+4}$ \\
\hline $\mathrm{CPE} \quad\left(\mathrm{F} / \mathrm{cm}^{2}\right)$ & $8,74 \times 10^{-3}$ & $8,68 \times 10^{-4}$ & $3,69 \times 10^{-4}$ & $5,43 \times 10^{-4}$ \\
\hline $\mathrm{n}$ & 0,810 & 0,927 & 0,691 & 0,907 \\
\hline \multicolumn{5}{|c|}{ CON TRATAMIENTO TÉRMICO } \\
\hline $\mathrm{R}_{\text {solución }}\left(\Omega . \mathrm{cm}^{2}\right)$ & 17,6 & 18,0 & 19,1 & 17,9 \\
\hline $\mathrm{R}_{\text {oxido }}\left(\Omega . \mathrm{cm}^{2}\right)$ & $3,63 \times 10^{+2}$ & $2,16 \times 10^{+2}$ & $2,75 \times 10^{+2}$ & $5,42 \times 10^{+2}$ \\
\hline $\mathrm{CPE} \quad\left(\mathrm{F} / \mathrm{cm}^{2}\right)$ & $1,88 \times 10^{-2}$ & $2,28 \times 10^{-2}$ & $1,05 \times 10^{-2}$ & $1,31 \times 10^{-2}$ \\
\hline $\mathrm{n}$ & 0,829 & 0,832 & 0,845 & 0,789 \\
\hline
\end{tabular}

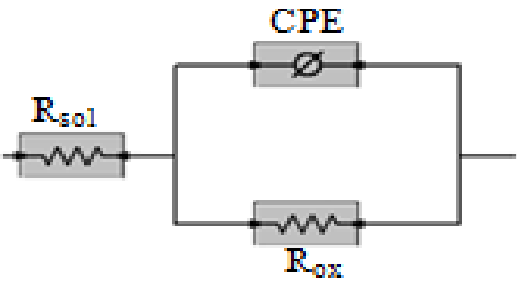

Figura 8: Circuito eléctrico equivalente.

Las respuestas de EIS para las películas de $\mathrm{ZnO}$ mostraron un comportamiento capacitivo dominante en todo el rango de frecuencias considerando en esta investigación, asociado con la presencia de un elemento de fase constante, con coeficiente de identidad cercano a la unidad.

Un coeficiente de identidad " $n$ " del elemento de fase constante cercano a uno, significa que las películas anódicas tienen un comportamiento capacitivo, la ligera disminución de este valor de " $n$ " se puede atribuir a heterogeneidades superficiales en las películas de $\mathrm{ZnO}$, en la Figura 4 se observa que las películas presentan una morfología poco homogénea a causa de los aglomerados de partículas que se forman sobre todo a potenciales elevados en ausencia de agitación del medio electrolítico, sin embargo, las películas se notan compactas por lo que se puede asumir un comportamiento capacitivo ideal.

Asumiendo un comportamiento capacitivo puro de los recubrimientos obtenidos, es factible estimar los espesores relativos de las películas sintetizadas a diferentes voltajes a partir de los valores de CPE obtenidos mediante la aproximación a circuitos eléctricos equivalentes de las medidas de EIS, aplicando la Ecuación 7 del capacitor de platos paralelos [13].

$$
C=\frac{\varepsilon \varepsilon_{0} a}{d}
$$

Donde $\varepsilon_{0}$ representa la constante dieléctrica en vacío, $\mathcal{E}$ es la constante dieléctrica relativa de las películas de óxido anodizadas, $a$ es el área del electrodo y $d$ es el espesor de la capa dieléctrica. En la Tabla 2 se presenta una comparación de los espesores relativos de las películas de $\mathrm{ZnO}$ sin tratamiento térmico y con tratamiento térmico. Según la Ecuación 7, los espesores de las películas son inversamente proporcionales a la capacitancia del sistema.

Tabla 2: Comparación de propiedades de las películas obtenidas.

\begin{tabular}{l|l|l}
\hline PROPIEDAD & SIN TRATAMIENTO TÉRMICO & CON TRATAMIENTO TÉRMICO \\
\hline Espesor & $30 \mathrm{~V} \approx 40 \mathrm{~V}>20 \mathrm{~V}>10 \mathrm{~V}$ & $10 \mathrm{~V} \approx 20 \mathrm{~V} \approx 30 \mathrm{~V} \approx 40 \mathrm{~V}$ \\
\hline Compactación & $20 \mathrm{~V}>40 \mathrm{~V}>10 \mathrm{~V} \approx 30 \mathrm{~V}$ & $10 \mathrm{~V} \approx 20 \mathrm{~V} \approx 30 \mathrm{~V} \approx 40 \mathrm{~V}$ \\
\hline
\end{tabular}


En base a los parámetros analizados en las películas de $\mathrm{ZnO}$ sin tratamiento térmico, se demostró que al aplicar potenciales mayores durante su formación, se obtuvieron capas de mayor espesor y mayor resistencia, en tanto que, a potenciales menores las capas tendían a ser más delgadas y menos resistivas. Esta diferencia de espesores se relaciona con las diferencias en las dimensiones de las partículas de ZnO que constituyen dichos recubrimientos. A potenciales bajos, las partículas de $\mathrm{ZnO}$ presentan un diámetro menor que las partículas obtenidas a potenciales mayores, sin embargo, a potenciales bajos las películas son mucho más delgadas y compactas, y su morfología es también más homogénea.

La revisión bibliográfica postula que los tamaños de partículas obtenidas son directamente proporcionales al potencial aplicado [4] lo que induce a pensar que a potenciales mayores los tamaños de las partículas serán mayores y por ende los espesores de las películas obtenidas también lo serán, sin embargo, la evidencia demuestra que a un potencial de $40 \mathrm{~V}$ se obtuvo un recubrimiento de espesor inferior al obtenido tras aplicar un potencial igual a 30V. Si se compara la Figura 4.b y 4.c se observa que no existe una diferencia evidente en los tamaños de partículas de $\mathrm{ZnO}$, pero si hay una marcada diferencia en la morfología de las películas, a $40 \mathrm{~V}$ los aglomerados de $\mathrm{ZnO}$ se distribuyen con mayor irregularidad respecto a las películas obtenidas a $30 \mathrm{~V}$.

Pese a que la estimación de los espesores relativos de las películas de óxido de zinc se realizó bajo el supuesto de un comportamiento capacitivo ideal, el coeficiente de identidad " $n$ " asociado al elemento de fase constante CPE difiere levemente de la unidad, esta disminución del valor de " $n$ " puede asociarse a la presencia de una película porosa o bien a heterogeneidades superficiales de las películas [10]. Las micrografías de las películas de $\mathrm{ZnO}$ obtenidas a diferentes potenciales presentadas en la Figura 3, evidencian que las películas obtenidas no son porosas, sino que la disminución del valor del coeficiente de identidad podría estar asociada a las acumulaciones desordenadas de las partículas en toda la superficie. En función de los valores adoptados por "n" se demostró que la película resultante del anodizado a $30 \mathrm{~V}$ presentó una mayor heterogeneidad en su superficie, mientras que las capas obtenidas a $10 \mathrm{~V}, 20 \mathrm{~V}$ y $40 \mathrm{~V}$ resultaron ser más compactas.

\subsection{Influencia del tratamiento térmico en las propiedades electroquímicas de las películas}

Por microscopía electrónica se observa que las nanopartículas de $\mathrm{ZnO}$ obtenidas durante el proceso de anodizado se distribuyen en toda la superficie del sustrato adoptando una morfología desordenada, lo que indica que en las condiciones anodizadas de obtiene una cristalización del $\mathrm{ZnO}$ de baja calidad, este efecto es aún más notorio cuando el material se oxida a potenciales elevados. Para controlar la cristalinidad de las estructuras se aplicó un tratamiento térmico a los anodizados obtenidos, sometiendo a las mismas a un recocido a $250^{\circ} \mathrm{C}$ durante dos horas. A nivel macroscópico, se observó que las películas tratadas térmicamente presentaron una coloración blanca, homogénea y uniforme en toda su extensión superficial, lo que indica que las películas tienen una composición uniforme [3, 14-17].

La influencia del tratamiento térmico en las propiedades electroquímicas de los anodizados se evaluó por EIS. Para las películas de $\mathrm{ZnO}$ tratadas térmicamente, los datos experimentales obtenidos por EIS también se ajustaron a un circuito equivalente simple semejante al de la Figura 8 con valores de los coeficientes de identidad cercanos a uno, en este caso, los valores de n para las películas obtenidas a diferentes voltajes son aproximadamente iguales en comparación con las que no se trataron térmicamente, es decir, que las estructuras de las películas tratadas térmicamente presentan menos heterogeneidades que las películas sin tratamiento térmico y por otra parte, no existe diferencia evidente entre las heterogeneidades estructurales de las películas obtenidas a diferentes voltajes con tratamiento térmico, ya que su valor de coeficiente de identidad es aproximadamente el mismo para todas las probetas.

En la Tabla 1 se presentan los valores de los elementos constituyentes de los circuitos equivalentes que se ajustan a los datos obtenidos por medidas de EIS para los anodizados obtenidos a 10V, 20V, 30V y $40 \mathrm{~V}$; sin y con tratamiento térmico. Se puede observar que las propiedades electroquímicas de las películas difieren entre sí en función del valor de potencial aplicado cuando las probetas no son tratadas térmicamente, es decir que en ausencia de un tratamiento térmico los valores de las resistencias de las películas, de CPE y en consecuencia los espesores de las mismas son diferentes: a $30 \mathrm{~V}$ y $40 \mathrm{~V}$ se obtuvieron películas de mayor espesor y mayor resistencia en comparación con las obtenidas a $20 \mathrm{~V}$ y $10 \mathrm{~V}$, mientras que las películas obtenidas a $20 \mathrm{~V}$ presentaron una compactación mayor respecto a las obtenidas a $40 \mathrm{~V}$, y ésta a su vez, resultó más compacta que las películas obtenidas a $10 \mathrm{~V}$ y $30 \mathrm{~V}$.Sin embargo, cuando las películas son tratadas térmicamente los valores de las resistencias de las películas obtenidas a $10 \mathrm{~V}, 20 \mathrm{~V}, 30 \mathrm{~V}$ y $40 \mathrm{~V}$ son aproximadamente iguales, como lo también lo son los valores de n y de CPE, lo que sugiere que tras aplicar un tratamiento térmico las propiedades electroquímicas de las películas se homogeneizan y dejan de ser función del potencial aplicado durante su síntesis. Todas las películas tratadas térmicamente exhiben propiedades electroquímicas similares y son de igual espesor, independientemente de las condiciones de anodizado. 
Por otra parte, si se comparan las propiedades de las películas antes y después de aplicar el tratamiento térmico, se observa que el recocido produjo una disminución significativa de la resistencia eléctrica de los óxidos, acompañado con un aumento notorio en la capacitancia del sistema. Como el espesor de la película se óxido es inversamente proporcional a la capacitancia el sistema, el aumento del valor de CPE tras aplicar el tratamiento térmico implicó una disminución significativa de los espesores de las películas como así también su compactación. Los tratamientos térmicos realizados a temperaturas cercanas o inferiores al punto de fusión del Zinc producen reordenamientos estructurales en las regiones superficiales de las películas, sin embargo, a esas temperaturas lo fenómenos de mayor importancia son los de liberación de agua del $\mathrm{Zn}(\mathrm{OH})_{2}$ remanente en la superficie y su transformación en $\mathrm{ZnO}$ [3,9], la pérdida de moléculas de agua de las estructuras contribuyen en gran medida a la compactación y disminución del espesor de la película anódica, como así también provocan la disminución de la resistencia de las mismas.

\section{CONCLUSIONES}

Se sintetizaron exitosamente películas de $\mathrm{ZnO}$ por anodizado potenciostático en solución diluida de $\mathrm{NaOH}$. Las propiedades de las películas obtenidas se investigaron antes y después de aplicarse un tratamiento térmico a $250^{\circ} \mathrm{C}$ durante dos horas, mediante estudios electroquímicos, implementando la técnica de Espectroscopía de Impedancia Electroquímica (EIS) y de Microscopía Electrónica de Barrido (SEM).

Mediante la aproximación a circuitos equivalentes de los datos obtenidos por EIS, se demostró que las películas de $\mathrm{ZnO}$ presentaron un comportamiento capacitivo puro tanto para los especímenes sin tratamiento térmico como para las películas tratadas térmicamente, el mismo se asoció a la presencia de un elemento de fase constante con un coeficiente de identidad " $n$ ", levemente menor que la unidad. Asumiendo la presencia de un capacitor ideal, se estimaron los espesores de las películas obtenidas aplicando la ecuación del capacitor de platos paralelos, demostrándose que a mayores potenciales se obtuvieron capas de mayor espesor y poca homogeneidad en las películas sin tratamiento térmico. Por otra parte, los estudios de EIS demostraron que tras aplicar un tratamiento térmico en las películas anódicas se produjeron reordenamientos estructurales en las regiones superficiales de las películas, provocando una disminución de la resistencia de las mismas, además de que por encima de los $125^{\circ} \mathrm{C}$ las partículas de $\mathrm{Zn}(\mathrm{OH})_{2}$ sufrieron cambios por liberación de agua y transformación en $\mathrm{ZnO}$, lo que desembocó en una compactación de las películas y en el aumento de la capacitancia del sistema.

Las imágenes SEM evidenciaron la influencia del potencial aplicado en las características de las partículas de $\mathrm{ZnO}$ obtenidas. Al aplicar potenciales bajos se obtuvieron partículas de $\mathrm{ZnO}$ pequeñas, que se distribuían de manera homogénea en toda la superficie del sustrato. Mientras que a potenciales mayores, los diámetros de las partículas encontrados fueron significativamente mayores y su disposición más desordenada. No obstante, tras aplicar un tratamiento térmico, se encontró que no existen diferencias en las propiedades electroquímicas de las películas obtenidas a diferentes potenciales.

\section{AGRADECIMIENTOS}

Los autores agradecen al FONCyT-ANPCyT por el subsidio recibido para financiar la presente investigación (PICT-2012-2952) y al Consejo Interuniversitario Nacional (CIN) por la beca otorgada.

\section{BIBLIOGRAFÍA}

[1] YANG, L.L., Synthesis and Characterization of ZnO nanostructures, Physical Electronic Division Department of Science and Technology, Linköping University, SE-601 7, Norrköping, Suecia, 2010.

[2] PÉREZ TABORDA, J. A., et al. "Películas nanoestructuradas de óxido de zinc (ZnO)", Scientia et Technica, v. 2, n. 39, pp. 416-421, Sept. 2008.

[3] PREPELITA, P., et al., "Evolution of the properties of $\mathrm{ZnO}$ thin films subjected to heating treatments", Thin Solid Films, ELSEVIER, Science Direct, v. 520, n. 14, pp. 4689-4693, 2012.

[4] HUANG, G.S., WU, X.L., CHENG, Y.C. "Fabrication and characterization of anodic ZnO nanoparticles", Applied Physicd A. Materials Science \& Processing, v. 86, n. 4, pp. 463-467, Dic. 2007.

[5] SHETTY, A., NANDA, K.K., "Synthesis of zinc oxide porous structure by anodization with water as an electrolyte", Applied Physicd A. Materials Science \& Processing, v. 109, n. 1, pp. 151-157, 2012.

[6] GOH, H., ADNAN, R., FARRUKH, A., “ZnO nanoflake arrays prepared via anodization and their performance in the photodegradation of methyl orange", Turk J Chem TUBITAK, v. 35, n.3, pp. 375-391, 2011. 
[7] VARNAMKHASTI, M., FALLAH, H., ZADSAR, M., "Effect of heat treatment on characteristics of nanocrystalline ZnO films by electron beam evaporation", Vacuum. ELSEVIER, v. 86,n. 7, pp. 871-875, 2012.

[8] CHANG, J., LIN, C., LIAO, C.M., et al., "Effect of heat- treatment on characteristics of anodized aluminium oxide formed on ammonium adipate solution", Journal of Electrochemecal Society, v. 151, n.3, pp. 188194, Feb. 2004.

[9] GARZÓN-RAMOS, D., CANO PLATA, E., VARGAS-HERNÁNDEZ, C., "Estudios por impedancia eléctrica de nanoestructuras de ZnO", Scientia et technica, v. 18, n. 2,pp. 309-314, Agosto 2013.

[10] ORAZEM, M., TRIBOLLET, B., Electrochemecal Impedance Spectroscopy, 2 ed., New Jersey, John Wiley and Sons.

[11] BEEDRI, N., INAMDAR, Y., SAYYED, S.A. et al. "Growth of Zinc Oxide porous film via electrochemecal anodization using glycerol based electrolyte", Chemistry \& Chemecal Technology, v. 8, n.3, pp. 283286, 2014.

[12] PIRATOBA MORALES, U., CAMARGO, A., FLÓREZ, J., "Impedancia Electroquímica: Interpretación de diagramas típicos con circuitos equivalentes”, Dyna, v. 77, n. 164, pp. 69-75, 2010.

[13] GÓMEZ SANCHEZ, A., SCHREINER, W., DUFFÓ, G., et al., "Surface modification of titanium by anodic oxidation in phosphoric acid at low potentials. Part1. Structure, electronic properties and thickness of the anodic films", Surf. Interface Anal, v. 45, n. 6, pp. 1037-1046, 2012.

[14] ZHAO, J., WANG, X., D., et al. "Controllable growth of zinc oxide nanosheets and sunflower structures by anodization method", Materials Chemistry and Physics, ELSEVIER, v. 126, n. 3, pp. 555-559, 2011

[15] HE, S., ZHENG, M., YAO, L. et al. "Preparation and properties of nanostructures by electrochemical anodization method", Applied Surface Science, v. 256, n. 8, pp. 2557-2562, 2010.

[16] AOUN, Y., BENHAOUA, B., BENRAMACHE, S., et al., "Effect of annealing temperature on structural, optical, and electrical properties of zinc oxide $(\mathrm{ZnO})$ thin films deposited by spray pyrolysis technique", Optik, ELSEVIER, v. 126, n. 24, pp. 5407-5411, 2015.

[17] CAGLAR, Y., LLICAN, S., CAGLAR, M., et al., "Influence of heat treatment on the nanocrystalline structure of ZnO film deposited on p-Si", Journal of Alloys and Comounds, v. 481, n. 1, pp. 885-889, Abril. 2009. 